\title{
Mating system of Pachycereus pringlei: an autotetraploid cactus
}

\author{
DARLYNE A. MURAWSKI*, THEODORE H. FLEMING $\dagger$, KERMIT RITLAND $\ddagger$ \& J. L. \\ HAMRICK \\ Departments of Botany and Genetics, University of Georgia, Athens, GA 30602, †Department of Biology, University of \\ Miami, Coral Gables, FL 33124, USA and $\ddagger$ Department of Botany, University of Toronto, Toronto Canada, M5S3B2
}

\begin{abstract}
The mating system of the Mexican subdioecious columnar cactus, Pachycereus pringlei (Cardón), was examined by allozyme analysis. Tetrasomic patterns of inheritance were found for all polymorphic loci, indicating that the species is an autotetraploid. A model is presented that expands Ritland's (1990a) mixed mating model for autotetraploids to incorporate an arbitrary number of alleles per locus. This model is applied to the progeny arrays of several female and hermaphroditic individuals of $P$. pringlei. $P$. pringlei exhibited a complex breeding system; the multilocus estimate of outcross-

ing, $t_{m}$, for female cacti was 0.949 and for hermaphrodites was 0.301 . These results are discussed in the context of inbreeding depression and the evolution of the breeding system of this species.
\end{abstract}

Keywords: allozyme analysis, autotetraploidy, cactus, mating system, Mexico, Pachycereus.

\section{Introduction}

Naturally occurring autopolyploids have long been considered rare and maladaptive on the theoretical grounds that chromosome pairing difficulties during meiosis would prevent stable and reliable genetic transmission. Studies that used morphological traits to distinguish auto- and allopolyploid modes of speciation were often speculative as experimentally derived autopolyploids do not always resemble the parental diploid (Grant, 1981). However, a growing number of studies using genetic information in addition to cytological and morphological traits confirm that autotetraploids are more common than was originally thought. Species that have been shown to be autopolyploid or to have allozyme banding patterns that are consistent with autoploidy include alfalfa, Medicago sativa (Quiros, 1982), Antennaria media (Bayer et al., 1990), Heuchera micrantha (Ness et al., 1989), Maclura pomifera (Schnabel et al., 1991) and Dipteryx panamensis (Murawski \& Hamrick, unpublished data).

During an initial allozyme survey of three large, columnar cacti of the Sonoran desert (J. L. Hamrick \& T. H. Fleming, unpublished data), the Cardón cactus, Pachycereus pringlei, was found to have an unusually low proportion of homozygosity and both balanced

*Correspondence: Dr D. A. Murawski, Harvard University Herbaria, 22 Divinity Avenue, Cambridge, MA 02138, USA. and unbalanced heterozygotes at all of its loci, an observation that suggested autopolyploidy. Consistent with this observation was the haploid chromosome number of 22 reported for $P$. pringlei (Pinkava \& McLeod, 1971; Pinkava et al., 1973) which is double that of the majority of cacti including other members of Pachycereus (Katsgiris, 1952; Pinkava et al., 1977). P. pringle $i$ is also an unusual cactus because it is subdioecious with male, female, hermaphroditic and neuter individuals occurring at varying frequencies in its populations (T. H. Fleming, unpublished data).

In this paper allozyme assays of progeny arrays produced by controlled crosses are used to confirm the nature of polyploidy (auto vs. alloploidy). Additional progeny arrays derived from open-pollinated flowers are used to compare the outcrossing rates of female and hermaphroditic individuals. A multiallelic autotetraploid mating system model is developed to utilize the highly polymorphic allozyme loci found in Cardón.

\section{Materials and methods}

Cardón, Pachycereus pringlei, is a large columnar cactus (up to $15 \mathrm{~m}$ ) restricted to the coastal desert portions of the Mexican states of Sonora and Baja California. Its breeding system, which will be described in detail elsewhere, is cryptically subdioecious with four sexual types in the Bahia Kino region of Sonora. These 
types and their relative frequencies for 211 adult individuals in our main study area include male steriles ( 42 per cent), hermaphrodites ( 28 per cent), female steriles (28 per cent) and neuters (male and female steriles, 2 per cent). The Cardón flowering season lasts from late March through late May with a flowering peak in late April. Flowers open shortly after sunset and close by noon the next day. At night they are visited by the nectar and pollen-eating bat Leptonycteris curasoae and several species of small moths. Beginning at sunrise, flowers are visited by a variety of bird species and bees (primarily the exotic Apis mellifera). Pollinator exclusion experiments indicate that nocturnal and diurnal visitors are equally effective pollinators although the large, pollen and nectar-rich flowers are most strongly coevolved with bats (T. H. Fleming, Horner \& Tuttle, unpublished data).

\section{Study area and population samples}

Field aspects of this study were conducted between 15 April and 30 June 1989 and 1 April and 29 June 1990 near Bahia Kino, Sonora, Mexico $\left(28^{\circ} 50^{\prime} \mathrm{N}\right)$. This site is part of the Central Gulf Coast region of the Sonoran desert and is dominated by Bursera microphylla and two species of Jatropha (Shreve \& Wiggins, 1964). Other common plants include Larrea tridentata, Cercidium microphyllum, Olneya tesota and Fouquieria splendens. In addition to Pachycereus pringlei, three other columnar cactus species, Carnegia gigantea, Stenocereus thurberi and Lophocereus schotii are common in the area. Annual rainfall at Bahia Kino averages about $90 \mathrm{~mm}$; most rain falls in July and August. Topography in the area includes gravelly flatlands punctuated by rugged hills $200-450 \mathrm{~m}$ above sea level. Our main study area, located about $9 \mathrm{~km}$ northeast of Nuevo Bahia Kino, encompassed about 100 ha and contained 211 marked and sexed adult Cardóns.

To determine rates of self-fertilization by hermaphrodites in the absence of pollinators, four flowers on each of 14 individuals were bagged with bridal veil netting shortly before opening; the netting was removed the following afternoon after the flowers had closed. Half of the flowers were bagged on 30 April 1990 and the other half on 8 May. Seeds from the five surviving fruits (from three individuals) were collected in late June. These were then used to test progeny genotypic proportions against those expected given tetrasomic inheritance.

An initial survey of allozyme polymorphism was based on a sample of seeds from 14 open-pollinated individuals (eight females, six hermaphrodites) collected in 1989. To determine maternal genotypes for mating system analyses, flower bud tissue was collected from 14 females and 14 hermaphrodites on 10 May 1990. Tissue was refrigerated until it was airshipped on ice to the laboratory at the University of Georgia. To estimate outcrossing rates, on 17 June 1990 two fruits from open-pollinated flowers were collected from each of these individuals and their seeds were removed and air-dried. 17 June was early in the fruit ripening period and although care was taken to select the largest, ripest fruit from each plant, not all of these fruits contained mature, germinable seeds.

\section{Electrophoresis}

Seeds were germinated on moist filter paper in petri dishes inside growth chambers. Germination occurred within 1 week. Seedlings at the cotyledon stage (2 weeks old) were prepared for electrophoresis by crushing with the PVP extraction buffer of Mitton et al. (1979). Adult (bud) material was crushed in liquid nitrogen before adding the extraction buffer. Wicks containing seedling and adult material were sorted in an ultracold freezer $\left(-70^{\circ} \mathrm{C}\right)$ until needed for electrophoresis. Our goal was to assay 64 seeds from each plant (32 seeds from each fruit if two fruits were collected) although actual progeny numbers were reduced in some families because of poor germination.

Seven enzyme systems with eight loci were assayed. Additional polymorphic loci were detected but not assayed due to inconsistent resolution. The enzyme systems and locus designations are as follows: phosphoglucomutase (Pgm1, Pgm2), phosphoglucoisomerase (Pgi), alcohol dehydrogenase (Adhl), isocritrate dehydrogenase $(I d h)$, shikimate dehydrogenase $(S k d h)$, malate dehydrogenase $(M d h)$, aspartate amino transferase (Aat2). Adult genotypes were scored for PGM, PGI, $I D H$ and $S K D H$, and inferred from progeny genotypic proportions for $M D H$ and $A A T$. The buffer systems and staining recipes of Soltis et al. (1983) were employed: system 4 for $I D H$ and $S K D H$, system 7 for $M D H$ and $A A T$ and a modification of system 6 for $P G M, P G I$ and $A D H$.

\section{Data analysis}

Genotype frequencies resulting from the selfed crosses were compared with expected ratios based on tetrasomic inheritance. Expected ratios for balanced heterozygotes $(a a b b)$ were 1(aaaa): $8(a a a b)$ : $18(a a b b)$ : $8(a b b b): 1(b b b b)$ and for unbalanced heterozygotes were $1(a a a a$ or $b b b b): 2(a a a b$ or $a b b b): 1(a a b b)$. These ratios were determined by assuming that diploid gametes are produced. A given allele of the diploid pair is chosen at random without replacement with a proba- 
bility of $1 / 4$ and a second allele is chosen at random from the remaining genes with probability $1 / 3$ (Wendel \& Weeden, 1989).

Family genotypic data from open-pollinated fruits of females and hermaphrodites were analysed separately with a multiallelic extension of Ritland's (1990a) 'tetrat' program based on the procedures described in the Appendix. Multilocus and single-locus estimates of outcrossing rates were obtained for females and hermaphrodites. In addition, multilocus estimates were calculated for individual fruits of hermaphroditic plants.

The bootstrap method was used to determine the variance of the estimates by creating replicate data sets from progeny sampled randomly with replacement from the original data set. The distribution of the sample estimates determines the variance of estimates. This sampling was within families rather than between

a)
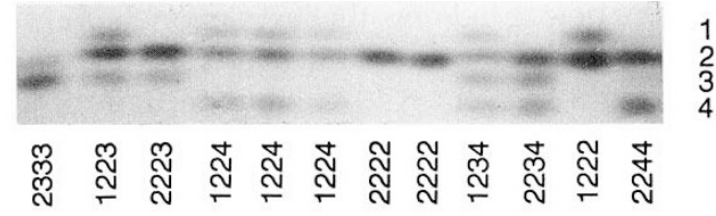

b)

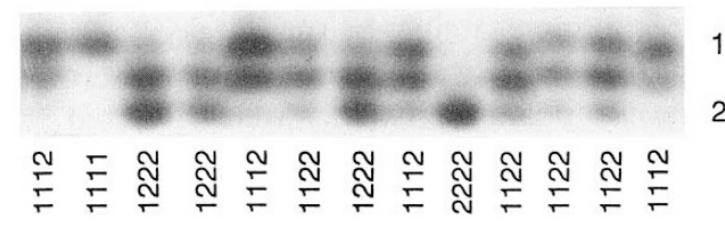

c)

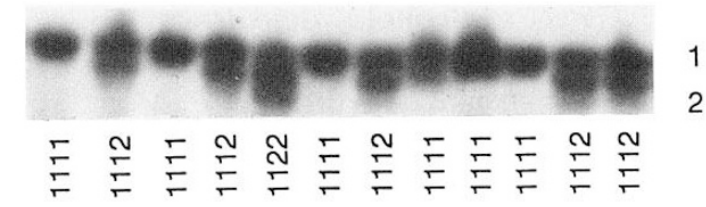

d)

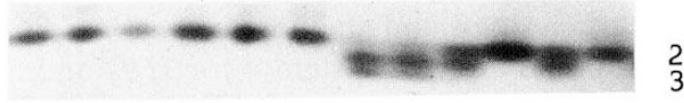

e)

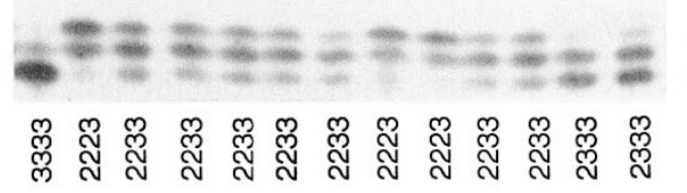

Fig. 1 Photographs of representative starch gels of Pachycereus pringlei. Numbers on the right side of the photographs designate alleles. Individual tetraploid genotypes are labelled beneath each lane. Note the presence of balanced and unbalanced heterozygotes at each locus. (a) $S k d h$, a monomer; (b) Aat2, a dimer; (c) $M d h 1$, a dimer; (d) Idh, a dimer; (e) $P g i$, a dimer. families because of the low number of families analysed. Consequently, standard errrors were not calculated for ovule allele frequencies. Fifty bootstraps were used for population and individual fruit estimates. Differences between ovule and pollen allele frequencies and between the pollen allele frequencies received by female and hermaphrodite plants were tested with the chi-squared statistic, where $\chi^{2}=N F_{\text {ST }}(\mathrm{a}-1)$; d.f. $=(\mathrm{a}-1) . F_{\mathrm{ST}}$ is a measure of the genetic diversity between the two groups (i.e. pollen vs. ovule or female vs. hermophrodites), $N$ is the sum of the gametes in the two groups.

\section{Results}

All polymorphic enzyme systems observed in $P$. pringlei showed evidence of the tetrasomic inheritance patterns indicative of autotetraploids (Fig. 1 and Table 1). Both balanced and unbalanced heterozygotes (corresponding to equal and unequal dosages of different alleles) were present. Owing to the isozyme diversity found in this plant, we observed as many as four allozyme alleles per locus in an individual plant (Fig. 1a). Additional polymorphic loci with similar tetrasomic patterns were found but not scored because of inconsistent resolution. There was no evidence of fixed heterozygotes as might be seen in allotetraploids.

Further evidence for autotetraploidy in $P$. pringle $i$ is provided by the genotypic frequencies of selfed crosses that did not differ significantly from the expected frequencies of $1: 8: 18: 8: 1$ for balanced heterozygous mothers and 1:2:1 for unbalanced heterozygotes (Table 1). These genotypic proportions in selfed progeny are indicative of independent chromosomal segregation (as in Weeden \& Wendel, 1989).

For most loci the allele frequencies of the pollen and ovule contributions to the progeny genotypes are similar (Table 2). There are, however, a few loci for which pollen and ovule allele frequencies are significantly different $(P<0.05)$. These include the $P g i$ locus for the female individuals and the Pgm2, Pgi and Aat2 loci for hermaphroditic individuals. Pollen allele frequencies received by females and hermaphrodites are also similar for the most part. Only the $S k d h$ and Aat2 loci are significantly different $(P<0.05)$ between hermaphroditic and female individuals.

The multilocus outcrossing estimate $\left(t_{\mathrm{m}}=0.301\right)$ for hermaphrodites was much lower than the estimate for females $\left(t_{\mathrm{m}}=0.949\right)$ (Table 3$)$. The average single locus estimate did not differ significantly from the multilocus estimate in females but was significantly lower in hermaphrodites $(P<0.05)$. This difference suggests the possibility of consanguineous matings but may also be due to correlated or non-random mating. Examining 
Table 1 Progeny genotypic frequencies of selfed crosses of Pachycereus pringlei. Expected progeny genotypic frequencies for balanced heterozygote mothers $(a a b b)$ assuming tetrasomic inheritance is the ratio $1: 8: 18: 8: 1$. For unbalanced heterozygotes (e.g. $a b b b$ and $a a a b$ ) the expected ratio is $1: 2: 1$

\begin{tabular}{|c|c|c|c|c|c|c|c|c|}
\hline \multirow[b]{2}{*}{ Locus } & \multirow{2}{*}{$\begin{array}{l}\text { Maternal } \\
\text { ID }\end{array}$} & \multirow{2}{*}{$\begin{array}{l}\text { Maternal } \\
\text { genotype }\end{array}$} & \multicolumn{5}{|c|}{ No. of offspring by genotype } & \multirow{2}{*}{$\begin{array}{l}\text { Significance } \\
\text { level of deviation } \\
\text { from expected } \\
\text { progeny frequency }\end{array}$} \\
\hline & & & aaaa & $a a a b$ & $a a b b$ & $a b b b$ & $b b b b$ & \\
\hline Aat2 & 634 & $a a b b$ & 2 & 15 & 37 & 8 & 1 & n.s. \\
\hline Aat2 & 666 & $a a b b$ & 1 & 17 & 33 & 12 & 1 & n.s. \\
\hline Aat2 & 623 & $a a b b$ & 4 & 1 & 12 & 4 & 0 & n.s. \\
\hline$P g i$ & 634 & $a a b b$ & 1 & 16 & 34 & 10 & 2 & n.s. \\
\hline$P g i$ & 666 & $a a b b$ & 1 & 18 & 32 & 11 & 1 & n.s. \\
\hline Pgm1 & 666 & $a a b b$ & 2 & 14 & 22 & 12 & 7 & n.s. \\
\hline$S k d h$ & 634 & $a b b b$ & & & 21 & 27 & 14 & n.s. \\
\hline Adh1 & 634 & $a a a b$ & 10 & 15 & 8 & & & n.s. \\
\hline Adh1 & 623 & $a a a b$ & 7 & 9 & 5 & & & n.s. \\
\hline
\end{tabular}

individual fruits of hermaphroditic mothers, the multilocus outcrossing estimates varied extensively from complete selfing to 72 per cent outcrossing. Heterogeneity in outcrossing rates was also found among fruits of the same plant, most notably 3 per cent and 52 per cent in plant no. 614 .

The two-locus fixation index in the pollen pool was positive for both females $(F=0.118 \pm 0.016)$ and hermaphrodites $(F=0.184 \pm 0.040)$, indicating a deficiency of heterozygous gametes compared with random expectations.

\section{Discussion}

Most of the quantitative data on plant mating systems is based on diploid species or on ancient polyploid species with diploid gene expression (see Schemske \& Lande, 1985 for a review of this literature). The absence of mating system analyses of polyploids is largely because the variety and complexity of segregation patterns in polyploids make quantitative estimates of the mating system difficult. It is only recently that algorithms have been developed to analyse the mating system of species with tetrasomic inheritance (Barrett \& Shore, 1987; Ritland, 1990a). As far as we are aware this is the first application of procedures that jointly infer selfing rate and parameters of maternal and paternal parentage under tetrasomic inheritance. In particular, tetrasomy provides an unique opportunity to infer correlations between paternal alleles, as paternal gametes possess two alleles per locus. The methods developed in the Appendix also represent a modification of the original algorithms to include more than two alleles per locus, a significant analytical advance for allele-rich tetraploid species such as $P$. pringlei.

The most striking result of our analysis is the significant difference in the estimates of outcrossing for female and hermaphroditic individuals. Females, as expected, had estimated rates of outcrossing that were close to $1.0(0.949 \pm 0.019)$. In sharp contrast, individuals with bisexual flowers had much lower multilocus estimates of outcrossing $(0.301 \pm 0.023)$ indicating that the majority of seeds from bisexual flowers result from self-pollination. In the apparent absence of a selfincompatibility system there is nothing in the morphology of the bisexual flower to prevent selfing. The stigma is placed at the same height as the abundant anthers. It is thus inevitable that large 'messy' pollinators such as $L$. curasoae would scatter considerable autogamous pollen onto the stigma.

It is also possible that additional inbreeding may result from the mating of related individuals. A lower single locus estimate of outcrossing $(0.238$ for hermaphrodites) relative to the multilocus estimate ( 0.301 for hermaphrodites) is usually indicative of biparental inbreeding (Brown et al., 1990). However, as we have independent estimates of outcrossing for females and bisexual individuals, we can calculate the level of biparental inbreeding more directly based on deviation of the mating system estimates of the females from unity (Sun \& Ganders, 1988). Because the mean single locus (0.979) and the multi-locus estimate (0.949) are only marginally significantly different from 1.0 we conclude that biparental inbreeding plays a relatively small role in the breeding system of this species. This result is consistent with what is known of the natural history of $P$. pringlei. Dense populations of this 
Table 2 Allele frequency estimates for pollen (with S.E.) and ovules of females and hermaphroditic Pachycereus pringlei. Standard errors are not given for ovule allele frequencies as bootstrap resampling was done within families due to the small number of families

\begin{tabular}{|c|c|c|c|c|}
\hline \multirow[b]{2}{*}{ Group } & \multirow{2}{*}{$\begin{array}{l}\text { Locus } \\
(n \text { seeds })\end{array}$} & \multirow{2}{*}{$\begin{array}{l}\text { Allele } \\
\text { designation }\end{array}$} & \multicolumn{2}{|c|}{ Allele frequency } \\
\hline & & & Pollen pool & Ovule pool \\
\hline \multirow[t]{24}{*}{ Females } & \multirow[t]{2}{*}{$\operatorname{Pgml}(663)$} & 1 & $0.988(0.003)$ & 0.994 \\
\hline & & 2 & $0.012(0.003)$ & 0.006 \\
\hline & \multirow[t]{5}{*}{$\operatorname{Pgm} 2(684)$} & 1 & $0.014(0.004)$ & 0.021 \\
\hline & & 2 & $0.577(0.015)$ & 0.550 \\
\hline & & 3 & $0.289(0.014)$ & 0.355 \\
\hline & & 4 & $0.096(0.007)$ & 0.086 \\
\hline & & 5 & $0.011(0.003)$ & 0.008 \\
\hline & \multirow[t]{4}{*}{$\operatorname{Pgi}(698)$} & 1 & $0.001(0.001)$ & 0.000 \\
\hline & & 2 & $0.538(0.016)$ & 0.488 \\
\hline & & 3 & $0.458(0.016)$ & 0.511 \\
\hline & & 4 & $0.001(0.001)$ & 0.000 \\
\hline & \multirow[t]{2}{*}{$\operatorname{Adhl}(691)$} & 1 & $0.955(0.006)$ & 0.968 \\
\hline & & 2 & $0.042(0.006)$ & 0.032 \\
\hline & \multirow[t]{2}{*}{$\operatorname{Idh}(700)$} & 1 & $0.871(0.009)$ & 0.917 \\
\hline & & 2 & $0.137(0.009)$ & 0.083 \\
\hline & \multirow[t]{5}{*}{$S k d h(691)$} & 1 & $0.083(0.009)$ & 0.086 \\
\hline & & 2 & $0.590(0.017)$ & 0.594 \\
\hline & & 3 & $0.167(0.013)$ & 0.185 \\
\hline & & 4 & $0.130(0.010)$ & 0.134 \\
\hline & & 5 & $0.002(0.002)$ & 0.001 \\
\hline & \multirow[t]{2}{*}{$\operatorname{Mdhl} 1702)$} & 1 & $0.932(0.008)$ & 0.945 \\
\hline & & 2 & $0.068(0.008)$ & 0.055 \\
\hline & \multirow{2}{*}{ Aat2(679) } & 1 & $0.518(0.019)$ & 0.511 \\
\hline & & 2 & $0.482(0.019)$ & 0.489 \\
\hline \multirow[t]{23}{*}{ Hermaphrodites } & \multirow[t]{2}{*}{$\operatorname{Pgm} 1(512)$} & 2 & $0.000(0.000)$ & 0.000 \\
\hline & & 3 & $1.000(0.000)$ & 1.000 \\
\hline & \multirow[t]{5}{*}{$\operatorname{Pgm} 2(508)$} & 1 & $0.000(0.000)$ & 0.004 \\
\hline & & 2 & $0.608(0.034)$ & 0.480 \\
\hline & & 3 & $0.366(0.034)$ & 0.388 \\
\hline & & 4 & $0.043(0.019)$ & 0.128 \\
\hline & & 5 & $0.000(0.000)$ & 0.000 \\
\hline & \multirow[t]{3}{*}{$\operatorname{Pgi}(512)$} & 2 & $0.498(0.037)$ & 0.430 \\
\hline & & 3 & $0.502(0.037)$ & 0.559 \\
\hline & & 4 & $0.000(0.000)$ & 0.011 \\
\hline & \multirow[t]{2}{*}{$\operatorname{Adhl}(496)$} & 2 & $0.916(0.022)$ & 0.909 \\
\hline & & 3 & $0.093(0.022)$ & 0.091 \\
\hline & \multirow[t]{2}{*}{$I d h(512)$} & 2 & $0.884(0.021)$ & 0.904 \\
\hline & & 3 & $0.138(0.021)$ & 0.096 \\
\hline & \multirow[t]{5}{*}{$S k d h(495)$} & 1 & $0.203(0.036)$ & 0.227 \\
\hline & & 2 & $0.520(0.037)$ & 0.476 \\
\hline & & 3 & $0.237(0.026)$ & 0.216 \\
\hline & & 4 & $0.054(0.016)$ & 0.080 \\
\hline & & 6 & $0.004(0.003)$ & 0.001 \\
\hline & \multirow[t]{2}{*}{$M d h 1(512)$} & 1 & $0.983(0.007)$ & 0.998 \\
\hline & & 2 & $0.017(0.007)$ & 0.002 \\
\hline & \multirow[t]{2}{*}{ Aat2(492) } & 1 & $0.547(0.037)$ & 0.469 \\
\hline & & 2 & $0.453(0.037)$ & 0.531 \\
\hline
\end{tabular}


Table 3 Multilocus and average single-locus outcrossing estimates for females and hermaphroditic individuals of Pachycereus pringlei. Multilocus estimates are also given for individual hermaphroditic cacti by fruit

\begin{tabular}{llllll}
\hline $\begin{array}{l}\text { Population or } \\
\text { individual }\end{array}$ & $n$ & $t_{\mathrm{m}}$ & $($ S.E. $)$ & $t_{\mathrm{s}}$ & (S.E.) \\
\hline Females & 627 & 0.949 & $(0.019)$ & 0.979 & $(0.023)$ \\
Hermaphrodites & 565 & 0.301 & $(0.023)$ & 0.238 & $(0.021)$ \\
Individual (fruits ID) & & & & & \\
$614(1)$ & 32 & 0.03 & $(0.04)$ & & \\
$614(2)$ & 32 & 0.52 & $(0.09)$ & & \\
$621(1)$ & 32 & 0.19 & $(0.07)$ & & \\
$621(2)$ & 32 & 0.38 & $(0.09)$ & & \\
623 & 27 & 0.15 & $(0.07)$ & & \\
630 & 64 & 0.72 & $(0.07)$ & & \\
634 & 25 & 0.43 & $(0.11)$ & & \\
638 & 64 & 0.18 & $(0.05)$ & & \\
$646(1)$ & 16 & 0.08 & $(0.07)$ & & \\
$646(2)$ & 48 & 0.17 & $(0.08)$ & & \\
663 & 52 & 0.26 & $(0.10)$ & & \\
$666(1)$ & 32 & 0.18 & $(0.09)$ & & \\
$666(2)$ & 32 & 0.00 & $(0.00)$ & \\
\end{tabular}

species consist of reproductive individuals separated by distances of $10-30 \mathrm{~m}$. Leptonycteris curasoae is also an important agent for seed dispersal. These strong flying animals have an average seed passage time of about $30 \mathrm{~min}$ (V. Sosa, unpublished data), making it unlikely that seeds would be deposited in the immediate vicinity of the fruiting adult from which they originated. Seeds defaecated together could be genetically related (Levin, 1981) but the relatively infrequent recruitment success of this cactus would most likely preclude the establishment of related individuals adjacent to one another.

One question that arises from our results is how the largely selfed progeny of the hermaphroditic individuals could compete with outcrossed progeny derived from females. In other words, why does inbreeding depression acting on the progeny of the hermaphrodites not reduce the fitness of bisexual individuals and lead to their exclusion from the population? Yet, in this population and in others that have been surveyed $(T$. H. Fleming, unpublished data) hermaphroditic individuals makes up a substantial proportion (up to 67 per cent) of the reproductive individuals. The key to this question probably lies in the ploidy level of $P$. pringlei. With tetrasomic inheritance selfing leads to lower levels of individual homozygosity than with diploids and, as a result, there should be less inbreeding depression. With balanced heterozygotes $(a a b b)$ only $1 / 18$ of the progeny of a self-fertilization will be homozygous $(a a a a$ or $b b b b)$ rather than the $1 / 2$ expected for selfed heterozygous diploids. Progeny of selfed unbalanced heterozygotes ( $a a a b$ or $a b b b$ ) would have 25 per cent homozygous progeny, still significantly less than that for selfed diploids. Individuals heterozygous for three or more alleles at a locus would have even lower proportions of homozygous progeny. Thus, as most of the adults in the population are heterozygous, we conclude that the potential cost of self-fertilization for species with tetrasomic inheritance is much lower than that experienced by most diploid species.

The correlation between the two alleles within parental gametes was observed to be about $F=0.12$. In a mixed-mating allotetraploid population with selfing rate $s$ and random outcrossing rate $t=1-s$, this correlation changes among generations according to the recursion in the absence of selection (Ritland, 1990b). In this relation, $F^{\prime}$ denotes the correlation in the

$F^{\prime}=t\left(\frac{F}{3}\right)+s\left(\frac{1+3 F}{4}\right)$

gametes produced by the offspring (second generation). Note that in contrast to diploid inheritance, random outcrossing does not completely eliminate this correlation. If selfing rates are consistent among generations, at equilibrium

$F=\frac{3 s}{8-5 s}$.

In our study, the mean selfing rate was about 30 per cent ( 60 per cent male sterile with $s=0$ and 40 per cent hermaphrodite with $s=0.7$ ). If this adequately represents the selfing practised the last few generations, we expect $F=0.14$. Values of observed parental $F$ which are much less than that expected based on the selfing rate are indicative of selection against selfed progeny (which reduces parental $F$ ). In our study, the observed $F(0.12)$ was only slightly less than the expected $F(0.14)$, supporting the above arguments for reduced inbreeding depression in autotetraploids.

The subdioecious breeding system of $P$. pringlei, to our knowledge, is unique among the columnar cacti. Most cactus species have perfect flowers and have well developed self-incompatibility systems. Controlled selfed crosses of one species of Pachycereus, P. pectinaboriginum and two of the columnar cactus species that co-occur with $P$. pringlei, Carnegia gigantea (sagauro) and Stenocereus thurberi (organ pipe), failed to produce seed or fruit whereas similar crosses of $P$. pringlei produced abundant seeds (T. H. Fleming, unpublished data). Although the breeding system of all but one diploid species of Pachycereus is unknown, we can speculate that the diploid ancestor of $P$. pringlei, like other columnar cacti, was most probably a self- 
incompatible hermaphrodite. Immediately after its development as an autotetraploid, $P$. pringlei was probably also a self-incompatible hermaphroditic species. However, as selection pressures against inbreeding and, in particular, selfing should be much lower in autotetraploids, selection to maintain the selfincompatibility system may have been relaxed. Furthermore, for a species whose seeds may be dispersed long distances by bats there may have been positive selection for isolated individuals to produce seeds via selffertilization. This conclusion is supported by the observation of Levin (1983) that breakdown of selfincompatibility is the most common alteration of the reproductive system following chromosome doubling in plants.

Later mutations could have been introduced in established populations that produced male sterility (i.e. females). In established populations females may have somewhat higher fitnesses relative to hermaphrodites due to marginally more fit progeny and because less energy is expanded to produce pollen. At Bahia Kino, for example, females produce 1.6 times more seeds annually than hermaphrodites (T. H. Fleming, Buchmann and Tuttle, unpublished data). Genes influencing female sterility (i.e. males) probably arose at a later time as males are not as geographically widespread as are hermaphrodites or females (T. H. Fleming, unpublished data). We have no information concerning the genetic control of male or female sterility but the ratios seen in this population and in others are consistent with a two nuclear gene, dominantrecessive system. The presence in this population of functionally neuter individuals is consistent with this hypothesis.

\section{Acknowledgements}

Field assistance was provided by P. Horner, T. May, J. Debelak, C. Sahley, T. Finn, and L. Sechback. G. Nabhan kindly transported plant tissues back to the U.S.A. We also thank a reviewer for suggesting a clarification in the notation used in the Appendix. Financial support (to TF) was provided by the National Geographic Society (grants 4024-89, 4263-90), National Fish and Wildlife Foundation (grant 90-015) and a University of Miami General Research Support Award.

\section{References}

BARRETT, S. AND SHORE, J. S. 1987. Variation and evolution of breeding systems in the Turnera ulmifolia L. complex (Turneraceae). Evolution, 41, 340-354.

BAYER, R. J., RITLAND, K. AND PURDY, B. G. 1990. Evidence of partial apomixis in Antennaria media (Asteraceae:
Inuleae) detected by the segregation of genetic markers. Am. J. Bot., 77, 1078-1083.

BROWN, A. H. D., BURDON, J. J. AND JARosz, A. M. 1989. Isozyme analysis of plant mating systems. In: Soltis, D. Soltis, P. (eds), Isozymes in Plant Biology, Dioscorides Press, Portland, Oregon, pp. 73-86.

Grant, v. 1981. Plant Speciation. Columbia University Press, New York.

KATAGIRIS, S. 1952. Studies on the chromosome number in some Cactaceae species. Jap. J. Breeding, 1, 233-236.

LEvin, D. A. 1981. Dispersal versus gene flow in plants. Ann. Mo. Bot. Gard., 68, 233-253.

LEVIN, D. A. 1983. Polyploidy and novelty in flowering plants. Am. Nature, 122, 1-25.

MITTON, J. B., LINHART, Y. B., STURGEON, B. K. AND HAMRICK, J. L. 1979. Allozyme polymorphism detected in mature needle tissue of ponderosa pine, Pinus ponderosa Laws. J. Heredity, 70, 86-89.

NESS, B. D., SOLTIS, D. E. AND SOLT1S, P. A. 1989. Autopolyploidy in Heuchera micrantha (Saxifragaceae). Am. J. Bot., 76, 614-626.

PINKAVA, D. J., MCGILl, L. A. AND REEVES, T. 1977. Chromosome numbers in some cacti of western North America III. Bull. Torrey Bot. Club, 104, 105-110.

PINKAVA, D. J. AND MCLEOD, M. G. 1971. Chromosome numbers in some cacti of Western North America. Brittonia, 23, 171-176.

PINKAVA, D. J., MClEOD, M. G., MCGILL, L. A. AND BRoWN, R. C. 1973. Chromosome numbers in some cacti of Western North America II. Brittonia, 25, 2-9.

Quiros, C. F. 1982. Tetrasomic inheritance for multiple alleles in alfalfa. Genetics, 101, 117-127.

RITLAND, K. 1990a. A series of FORTRAN computer programs for estimating plant mating systems. J. Heredity, 81, 235-237.

RITLAND, K. 1990b. Inferences about inbreeding depression based on changes of the inbreeding coefficient. Evolution, 44, 1230-1241.

SCHEMSKE, D. W. AND LANDE, R. L. 1985. The evolution of selffertilization and inbreeding depression in plants. II. Empirical observations. Evolution, 39, 41-52.

SCHNABEL, A., LAUSHMAN, R. H. AND HAMRICK, J. L. 1991. Comparative genetic structure of two co-occurring tree species Maclura pomifera (Moraceae) and Gleditsia triacanthos (Leguminosae). Heredity, 67, 357-364.

SHREVE, F. AND WIGGINS, 1. L. 1964. Vegetation and Flora of the Sonoran Desert, vols 1 and 2. Stanford University Press, Stanford, California.

SOlTiS, D. E., HAUFleR, C. H., DARRow, D. C. AND GASTONY; G. J. 1983. Starch gel electrophoresis of ferns: a compilation of grinding buffers, gel and electrode buffers and staining schedules. Am. Fern. J., 73, 9-15.

SUN, M. AND GANDERS, F. R. 1988. Mixed mating systems of Hawaiian Bidens (Asteraceae). Evolution, 42, 516-527.

WEEDEN, N. F. AND WENDEL, J. F. 1989. Genetics of plant isozymes. In: Soltis, D. and Soltis, P. (eds), Isozymes in Plant Biology, Dioscorides Press, Portland, pp. 46-72. 


\section{Appendix}

\section{Progeny probabilities for multiallelic loci in tetraploids}

Because of the large number of possible genotypes at multiallelic tetraploid loci, we develop a general notation for calculating the conditional probabilities of observing progeny genotypes, given parental genotypes and mating events. These probabilities are the basic elements in mating system estimation. Previous workers have given probabilities for the diallelic case only (c.f. Barrett and Shore, 1987; Ritland, 1990a). Our approach is based on defining variables that indicate the similarity of alleles observed among parents and their progeny. Once these variables are defined for any specific data item, a single formula gives the required probabilities as functions of these indicator variables. This formula reflects the mechanics of gene transmission and is easily encoded in a computer program despite its apparent complexity.

Firstly, for ease of understanding and for comparison, we develop these probabilities for diploids. Consider a maternal parent with alleles $A_{i} A_{j}$ and her offspring with alleles $A_{m} A_{n}$. The subscripts denote alleles, and take on numerical values from one to some arbitrary number of alleles. For example, if $i=j=2$, the mother is homozygous for the second allele in the population. Define the indicator variables $\delta_{i m}, \delta_{j m}, \delta_{i n}$ and $\delta_{i n}$, where, for example, $\delta_{i m}=1$ if $i=m$; otherwise $\delta_{i m}=0$. These $\delta \mathrm{s}$ indicate the identity-by-state of alleles. The probability of observing progeny genotype $A_{m} A_{n}$, given parent $A_{i} A_{j}$ has selfed, is

$\operatorname{prob}\left(A_{m} A_{n} \mid A_{i} A_{j}\right.$, self $)$

$$
=\left(2-\delta_{m n}\right)\left(\frac{\delta_{i m}+\delta_{j m}}{2}\right)\left(\frac{\left(\delta_{i n}+\delta_{j n}\right)}{2}\right) .
$$

In this expression, $\delta_{i m} / 2$ is the probability, conditioned on the data and mating event, that allele $i$ is the parent gamete; it is divided by two because there are two possible parent gametes, each with prior probability $1 / 2$. The probabilities involving the second progeny allele $n$ enter as a product because its transmission is an independent event. The $2-\delta_{m n}$ term enters because the progeny genotypes $A_{m} A_{n}$ and $A_{n} A_{m}$ are classified together.

The probability of observing progeny genotype $A_{m} A_{n}$, given parent $A_{i} A_{j}$ has outcrossed, is

$\operatorname{prob}\left(A_{m} A_{n} \mid A_{i} A_{j}\right.$, outcross $)$

$$
=\left(2-\delta_{m n}\right)\left(\frac{1}{2}\left(\frac{\delta_{i m}+\delta_{j m}}{2}\right) p_{n}+\frac{1}{2}\left(\frac{\left(\delta_{i n}+\delta_{j n}\right)}{2}\right) p_{m}\right),
$$

where $p_{n}$ is the frequency of allele $n$ in the outcrossing pollen pool (or, the prior probability of obtaining $A_{n}$ from an outcross). The $1 / 2$ enters because there are two possible origins of the progeny genotype, each with prior probability $1 / 2: A_{m}$ derived from selfing and $A_{n}$ derived from outcrossing, vs. $A_{m}$ derived from outcrossing and $A_{n}$ derived from selfing.

For the case of tetraploids, we denote the maternal parent as $A_{i} A_{j} A_{k} A_{l}$ and her offspring as $A_{m} A_{n} A_{o} A_{p}$. In tetraploids, pairs of alleles are transmitted to gametes and there are three possible ways the four alleles $A_{m} A_{n} A_{o} A_{p}$ were obtained from parents: (1) $A_{m} A_{n}$ in one gamete and $A_{o} A_{p}$ in the other, (2) $A_{m} A_{o}$ in one gamete and $A_{n} A_{p}$ in the other, and (3) $A_{m} A_{p}$ in one gamete and $A_{n} A_{o}$ in the other.

The first step in finding progeny probabilities is to specify the probability of observing $\left(A_{m} A_{n}\right)$ gamete from an $A_{i} A_{j} A_{k} A_{l}$ parent:

$$
\begin{aligned}
& {\left[\left(\delta_{i m}+\delta_{j m}+\delta_{k m}+\delta_{l m}\right)\left(\delta_{i n}+\delta_{j n}+\delta_{k n}+\delta_{l n}\right)\right.} \\
& \left.\quad-\left(\delta_{i m} \delta_{i n}+\delta_{j m} \delta_{j n}+\delta_{k m} \delta_{k n}+\delta_{l m} \delta_{l n}\right)\right] \div 12 \\
& \quad=\frac{\Delta_{m n}}{12} .
\end{aligned}
$$

The subtracted term above arises because in the formation of gametes, the second allele is derived from the same tetrad as the first, e.g. alleles are sampled without replacement from parent genotypes. This quantity is divided by 12 because the first allele is drawn from a pool of four gametes (thus $1 / 4$ prior probability) while the second allele is drawn from a pool of three remaining gametes (thus $1 / 3$ prior probability), giving $1 / 12$ total prior probability. Considering all three pairs of diploid gametes, each with a prior probability of $1 / 3$, the probability of observing $A_{m} A_{n} A_{o} A_{p}$, given its parentage and that it was a self, is

$$
\begin{aligned}
& \operatorname{prob}\left(A_{m} A_{n} A_{o} A_{p} \mid A_{i} A_{j} A_{k} A_{l}, \text { self }\right) \\
& =\left(\frac{c}{3}\right)\left(\left(\frac{\Delta_{m n}}{12}\right)\left(\frac{\Delta_{o p}}{12}\right)+\left(\frac{\Delta_{m o}}{12}\right)\left(\frac{\Delta_{n p}}{12}\right)+\left(\frac{\Delta_{m p}}{12}\right)\left(\frac{\Delta_{n o}}{12}\right)\right),
\end{aligned}
$$

where

$$
\begin{gathered}
c=24-12\left(\delta_{m n}+\delta_{m o}+\delta_{m p}+\delta_{n o}+\delta_{n p}+\delta_{o p}\right) \\
+6\left(\delta_{m n} \delta_{o p}+\delta_{m o} \delta_{n p}+\delta_{m p} \delta_{n o}\right)+16 \\
\left(\delta_{m n o}+\delta_{m n p}+\delta_{m o p}+\delta_{n o p}\right)-33 \delta_{m n o p}
\end{gathered}
$$

(this $c$ term is due to classifying together equivalent progeny genotypes, such as $A_{1} A_{1} A_{1} A_{2}$ with $A_{1} A_{1} A_{2} A_{1}$, etc.). In this expression, the indicator nota- 
tion is extended to triplets and quadruplets of alleles: $\delta_{m n o}=1$ if $m=n=o ; \quad \delta_{m n o}=0$ otherwise, and $\delta_{\text {mnop }}=1$ if $m=n=o=p ; \delta_{\text {mnop }}=0$ otherwise.

To obtain the probability of a progeny derived from outcrossing, note that we condition upon six possible pairs of alleles derived from the maternal parent: $A_{m} A_{n}, A_{m} A_{o}, A_{m} A_{p}, A_{n} A_{o}, A_{n} A_{p}$, and $A_{n} A_{o}$, each with prior probability $1 / 6$. Given a certain pair of maternal alleles, the probability of observing a progeny is determined by the outcrossing pollen pool frequency for the remaining pair of putative paternal alleles. Thus, the probability of observing $A_{m} A_{n} A_{o} A_{p}$ given its parentage and that it was outcrossed is

$$
\begin{gathered}
\operatorname{prob}\left(A_{m} A_{n} A_{o} A_{p} \mid A_{i} A_{j} A_{k} A_{l} \text {, outcross }\right)= \\
\langle c / 6)\left[\left(\frac{\Delta_{m n}}{12}\right) p_{o p}\right. \\
+\left(\frac{\Delta_{m o}}{12}\right) p_{n p}+\left(\frac{\Delta_{m p}}{12}\right) p_{n o}+\left(\frac{\Delta_{n o}}{12}\right) p_{m p} \\
\left.\left.+\left(\frac{\Delta_{n p}}{12}\right) p_{m o}+\left(\frac{\Delta_{o p}}{12}\right) p_{m n}\right)\right],
\end{gathered}
$$

where $p$ s denote pollen gene frequencies (e.g. $p_{m n}$ is the frequency of alleles $m$ and $n$ in the paternal gamete pollen pool).

Interestingly, the inbreeding coefficient of parents enters into this frequency as $p_{m n}=p_{m} p_{n}\left(1-F_{p o l}\right)+$ $\delta_{m n} p_{m} F_{p o l}$ (the standard formula for genotypic proportions under inbreeding described by Wright's $F$ but for $F$ defined as the 'two-gene' inbreeding coefficient among paternal, diploid gametes, $\left.F_{p o l}\right)$ ). This means that in tetraploids, two-gene inbreeding coefficients of males can be estimated from progeny arrays and compared with two-gene coefficients of females also estimated from progeny arrays. (However, note that there are additional inbreeding coefficients in tetraploids, specifically the three- and four-gene coefficients (see Bayer et al., 1990) that cannot be estimated for males.) By contrast, in diploids, inbreeding coefficients of males can only be estimated by paternity analysis.

The selfing and outcrossing probabilities determine the total probability of observing an offspring as

$$
\begin{aligned}
& \operatorname{prob}\left(A_{m} A_{n} A_{o} A_{p} \mid A_{i} A_{j} A_{k} A_{l}\right)== \\
& \quad s \operatorname{prob}\left(A_{m} A_{n} A_{o} A_{p} \mid A_{i} A_{j} A_{k} A_{l}, \text { self }\right) \\
& \quad+t \operatorname{prob}\left(A_{m} A_{n} A_{o} A_{p} \mid A_{i} A_{j} A_{k} A_{l} \text {, outcross }\right),
\end{aligned}
$$

where $s$ is the prior selfing rate and $t$ the prior outcrossing rate. The multilocus extension of this formula is of the form

$$
\begin{aligned}
& \left.\left.\operatorname{prob}\left\langle G_{o f f}\right| G_{p a r}\right)=s \prod_{i} \operatorname{prob}\left\langle G_{o f f, i}\right| G_{p a r, i} \text {, self }\right\rangle \\
& \quad+t \prod_{i} \operatorname{prob}\left(G_{o f f, i} \mid G_{p a r, i}, \text { outcross }\right)
\end{aligned}
$$

where $G_{o f f, i}$ and $G_{p a r, i}$ denote offspring and parent genotypes, respectively, at locus $i$; the prob's are the single locus probabilities derived above. At this point, the standard procedure and assumptions for outcrossing estimation is applied (Ritland, 1990a). A program that implements this procedure is available from $K$. $R$. on receipt of a floppy disk or via e-mail (ritland@ botany.utoronto.ca). 